Published in final edited form as:

J Am Geriatr Soc. 2014 January ; 62(1): 25-39. doi:10.1111/jgs.12611.

\title{
The Impact of Tai Chi on Cognitive Performance in Older Adults: A Systematic Review and Meta-Analysis
}

\author{
Peter M. Wayne, PhD ${ }^{a, b}$, Jacquelyn N. Walsh, BS ${ }^{a, b}$, Ruth E. Taylor-Piliae, PhD, RN ${ }^{c}$, \\ Rebecca E. Wells, MD, MPH ${ }^{d}$, Kathryn V. Papp, PhD $^{b, e}$, Nancy J. Donovan, Nancy J. ${ }^{\text {b,f }}$, and \\ Gloria Y. Yeh, MD, MPH ${ }^{\mathrm{b}, \mathrm{g}}$ \\ aOsher Center for Integrative Medicine, Division of Preventive Medicine, Brigham and Women's \\ Hospital, Boston, MA \\ bHarvard Medical School, Boston, MA \\ ${ }^{c}$ College of Nursing, University of Arizona, Tucson, AZ \\ dDepartment of Neurology, Wake Forest School of Medicine, Winston-Salem, NC \\ eDepartment of Psychiatry, Massachusetts General Hospital, Boston, MA \\ ${ }^{f}$ Center for Alzheimer Research and Treatment, Departments of Psychiatry and Neurology, \\ Brigham and Women's Hospital, Boston, MA \\ gDivision of General Medicine and Primary Care, Beth Israel Deaconess Medical Center, Boston, \\ MA
}

\begin{abstract}
OBJECTIVES-Summarize and critically evaluate research on the effects of Tai Chi on cognitive function in older adults.

DESIGN-Systematic review with meta-analysis.

SETTING—Community and residential care.

PARTICIPANTS-Individuals aged 60 and over (with the exception of one study) with and without cognitive impairment.

MEASUREMENTS—Cognitive ability using a variety of neuropsychological testing.

RESULTS-Twenty eligible studies with a total of 2,553 participants were identified that met inclusion criteria for the systematic review: 11 of the 20 eligible studies were randomized
\end{abstract}

Corresponding Author: Peter M. Wayne, PhD, Brigham and Women's Hospital, Division of Preventive Medicine, 900

Commonwealth Avenue, 3rd fl, Boston, MA 02215, USA. Tel: 617-732-6271; fax: 617-731-3843, (pwayne@ partners.org). Alternate Corresponding Author: Gloria Y. Yeh, MD, MPH (gyeh@bidmc.harvard.edu).

Conflict of Interest: The editor in chief has reviewed the conflict of interest checklist provided by the authors and has determined that the authors have no financial or any other kind of personal conflicts with this paper.

Author Contributions: Peter M. Wayne conceived the idea for this study. In addition, Peter M. Wayne wrote the first draft, extracted data, and evaluated studies for methodological quality and risk of bias. Jacquelyn $\mathrm{N}$. Walsh contributed to the first draft and extracted data. Ruth E. Taylor-Piliae conducted meta-analyses and contributed to the manuscript. Gloria Y. Yeh extracted data, evaluated studies for methodological quality and risk of bias, and contributed to the manuscript. Rebecca E. Wells, Kathryn V. Papp, and Nancy J. Donovan all contributed to the manuscript. 
controlled trials (RCTs), 1 was a prospective non-randomized controlled study, 4 were prospective non-controlled observational studies, and 4 were cross-sectional studies. Overall quality of RCTs was modest, with 3 of 11 trials categorized as high risk of bias. Meta-analyses of outcomes related to executive function in RCTs of cognitively healthy adults indicated a large effect size when Tai Chi was compared to non-intervention controls (Hedge's $\mathrm{g}=0.90 ; \mathrm{p}=0.043$ ) and moderate effect size when compared to exercise controls (Hedge's $g=0.51 ; \mathrm{p}=0.003$ ). Meta-analyses of outcomes related to global cognitive function in RCTs of cognitively impaired adults, ranging from mild cognitive impairment to dementia, showed smaller but statistically significant effects when Tai Chi was compared to both non-intervention controls (Hedge's $\mathrm{g}=0.35 ; \mathrm{p}=0.004$ ) and other active interventions (Hedge's $g=0.30 ; p=0.002$ ). Findings from non-randomized studies add further evidence that Tai Chi may positively impact these and other domains of cognitive function.

CONCLUSION-Tai Chi shows potential to enhance cognitive function in older adults, particularly in the realm of executive functioning and in those individuals without significant impairment. Larger and methodologically sound trials with longer follow-up periods are needed before more definitive conclusions can be drawn.

\section{Keywords}

Tai Chi; cognitive function; executive function; mind-body exercise

\section{INTRODUCTION}

Age-related cognitive decline is a burgeoning public health concern worldwide. In the United States alone, approximately 5.4 million people over age 70 have mild cognitive impairment (MCI) and an additional 3.4 million have dementia. ${ }^{1}$ Between 2005 and 2009, the worldwide cost of dementia increased by $\sim 34 \% .^{2}$ The relative lack of pharmaceutical treatments to halt cognitive decline associated with aging has contributed to a growing interest in low-cost behavioral interventions for improving cognition.

The most well-researched behavioral intervention for cognitive functioning is physical activity. ${ }^{3}$ A recent meta-analysis of 16 prospective studies found that physical exercise reduced the relative risk of dementia by $28 \% .{ }^{4}$ Hypothesized mechanisms for the cognitive benefits of physical exercise include direct effects on the brain, such as increased vasculature and production of neurotrophic factors, which may promote neuronal repair, neuronal growth, and plasticity. ${ }^{5,6}$ Physical exercise may also mitigate risk factors for cognitive decline such as cardiovascular disease. Other behavioral interventions have attempted to harness factors which have been shown to be protective against cognitive decline in epidemiological research such as: high educational achievement ('brain training' interventions), social engagement, and stress reduction interventions (meditation and relaxation). ${ }^{7,8}$ Recent reviews suggest that multimodal interventions that include more than one behavioral or lifestyle intervention may have greater likelihood of influencing neurobiological mechanisms underlying cognitive decline, compared to any one activity alone. ${ }^{9}$

Tai Chi is an increasingly popular multimodal mind-body exercise that incorporates physical, cognitive, social, and meditative components in the same activity. ${ }^{10}$ For both 
conceptual and practical reasons, Tai Chi might be an effective intervention to slow cognitive decline in both healthy adults as well as in individuals with cognitive impairment. As a physical exercise, Tai Chi provides both moderate aerobic and agility/mobility training, which are each believed to impact cognitive function via unique neurophysiological pathways. ${ }^{11}$ Tai Chi also involves the learning of choreographed movement patterns, which may support visuospatial processing, processing speed, and episodic memory. As a mindbody exercise, Tai Chi includes training in sustained attentional focus and multi-tasking. One hypothesis for age-related cognitive decline is that the brain's attentional control is reduced and information processing becomes less efficient. Meditation has been shown to impact attention and executive functions by increasing the brain's ability to allocate attentional resources. ${ }^{12,13}$ The meditative component of Tai Chi may have direct benefits on enhancing attention and executive functions; it may also improve cognition indirectly by mitigating the known effects of anxiety and depression on cognition through stress-related pathways. ${ }^{14}$ Engaging in Tai Chi in a group setting may have further benefits on cognition by enhancing mood and coping skills through social interactions and support. Because Tai Chi may impact cognitive function via a diverse and potentially synergistic set of mechanistic pathways, it is plausible that it may offer benefits superior to interventions that target only single pathways (e.g., aerobic training or stress reduction alone).

Prior studies support the potential of Tai Chi to attenuate age-related declines in cardiovascular disease, ${ }^{15}$ balance, ${ }^{16}$ and emotional well-being. ${ }^{14}$ Importantly, these studies also suggest that Tai Chi is safe for older adults and an enjoyable activity with the potential for long-term adherence and exercise maintenance. Individual studies have reported on cognitive outcomes in different populations, but to our knowledge, there has not been a comprehensive systematic review examining Tai Chi's impact on age-related cognitive decline in older adults. Our objective was to conduct a systematic review and meta-analysis of research examining Tai Chi as an intervention to attenuate age-related cognitive decline across the spectrum from normal cognition to dementia. Other than Tai Chi, there were no limitations concerning the type or number of comparison groups included in each study or any restrictions on study type or design.

\section{METHODS}

\section{Literature search}

Electronic literature searches were conducted using PubMed/MEDLINE, Cumulative Index to Nursing and Allied Heath Literature (CINAHL), Excerpta Medica Database (Embase), and the Cochrane Library from inception through March 2013. The search terms were tai chi, tai ji, tai chi chuan, memory, cognition, cognitive decline, and brain function. Hand searches were performed of retrieved articles for additional references.

\section{Eligibility criteria}

We included available randomized controlled trials (RCTs), prospective non-randomized controlled studies (PNRCS), prospective non-controlled observational studies (PNCS), and cross-sectional studies (CSS) published in English, where cognitive measures were examined as outcomes in relation to a Tai Chi intervention in both cognitively impaired and 
unimpaired populations. Both because of the limited number of published RCTs to date and because this review is the first attempt to synthesize research evaluating Tai Chi for cognitive function, we have chosen to include a diverse set of study designs. To strike a balance between minimizing bias and being comprehensive, we restrict our meta-analytic methods to RCTs, and use tables and narrative methods to report on additional studies, as well as the degree to which they support or broaden findings from RCTs.

\section{Data extraction and synthesis}

Data were extracted by two reviewers (PW, JW) independently in a standardized manner. Data pertinent to: location, study design, duration, subject population, interventions, cognitive outcomes measured and all cognition-related results were summarized in Tables 1 and 2. Inclusion and exclusion of studies were reported in accordance with the Preferred Reporting Items for Systematic Reviews and Meta-Analyses guidelines. ${ }^{17}$

For RCTs only, we used meta-analytical methods to synthesize a subset of the most commonly reported outcomes. Comprehensive Meta Analysis Version 2.0 software was used to calculate effect sizes (i.e., Hedges' g) and the 95\% confidence intervals using random effects models. ${ }^{18}$ For studies among adults without cognitive impairment, we calculated the effect size for all executive function outcomes, with separate analyses for comparisons of Tai Chi with active (e.g., walking) and non-active (e.g., usual care) control interventions. No other outcomes in this population were measured as consistently as executive function, thus precluding pooled analyses. For studies among cognitively impaired adults, we calculated the effect size for global cognition, with separate analyses for comparisons of Tai Chi with active and non-active control interventions. Again, no other outcomes in this population were measured as consistently as global cognition, thus precluding pooled analyses.

An overall summary effect was calculated, using a random effects model to estimate the mean of the distribution of effects that could have been observed (e.g., different subjects or intervention protocols). Under the random effects model, the individual study weights are more balanced, thus the summary effect is more conservative. We also calculated the ratio of true heterogeneity to total observed variation using the $\mathrm{I}^{2}$ statistic, as $\mathrm{I}^{2}$ is not directly affected by the number of studies in the analysis. $\mathrm{I}^{2}$ is not scale dependent, but is expressed as a ratio ranging from $0-100 \%(25 \%=$ low, $50 \%=$ moderate, $75 \%=$ high heterogeneity $)$.

Results from non-RCTs were synthesized and summarized in narrative form.

\section{Risk of bias in individual studies}

Quality of methodology reported and associated risk of bias was assessed by two reviewers (PW, GY) independently using the 12 criteria (rating: yes, no, unclear) recommended by the Cochrane Back Review Group. ${ }^{19}$ When necessary, discrepancies were rechecked with a third reviewer and consensus achieved by discussion. Studies that met 6 of the 12 criteria and had no serious flaw (such as drop-out rate higher than 50\%, and/or statistically significant and clinically important baseline differences that were not accounted for in the analyses) were rated as having low risk of bias. Studies that met fewer than 6 criteria or had 
a serious flaw were rated as having high risk of bias. Methodological quality and associated risk of bias for all RCTs are summarized in Table 3.

\section{RESULTS}

\section{Literature search}

Figure 1 summarizes the flow of our literature search and selection process. Our search resulted in 195 studies. Eighty-three articles were excluded because they were duplicates. Twelve were further excluded: 4 were irrelevant to the search (e.g., author name "Tai"), 5 were not published in English; and 3 were not full text articles. Of the remaining 71 articles, 27 did not report original data, 4 did not utilize Tai Chi as an intervention, and $20 \mathrm{did}$ not report a cognitive function outcome.

\section{Study designs}

The 20 eligible studies identified by our search are summarized in Tables 1 and 2 . These studies include 11 RCTs, 1 PNRCS, 4 PNCS, and 4 CSS.

\section{Participant characteristics and study setting}

Table 1 includes 11 studies that evaluated the impact of Tai Chi on older adults that had no reported cognitive impairment. ${ }^{20-30}$ Table 2 includes 9 studies in populations that reported varying degrees of cognitive impairment, ranging from MCI to irreversible dementia., $91-39$ Eight studies were conducted in the United States, 7 in China, 3 in France, and 1 in both Japan and Vietnam. All but one study 22 focused on older adults with average ages above 60 . These included 12 studies in community dwelling individuals, 4 in residents of assisted living facilities, 1 in healthy volunteers, and 3 in patients from hospital-based settings.

\section{Intervention and control group characteristics}

Tai Chi interventions varied greatly in content, dosage, duration and intensity. Of the 16 prospective studies, 8 interventions were described as variations of Yang style Tai Chi, ${ }^{27-29,32-34,38,39} 3$ of Sun style, ${ }^{25,31,37}$ and 1 of Tai Chi Chih. ${ }^{23}$ In all cases, interventions were described as simplified, and/or included a subset of movements typically learned in traditional longer Tai Chi forms. One study integrated Tai Chi exercises within a multicomponent intervention including cognitive behavioral therapy and support group. ${ }^{9}$ Two studies did not provide details on the Tai Chi intervention. ${ }^{23,26}$

Individual sessions varied in duration from 20-60 minutes. The frequency of sessions varied from 1 to 4 times per week, with overall training programs lasting 10 weeks to 1 year. Two studies conducted a year-long observation with an induction or adoption phase and a maintenance phase. ${ }^{29,39}$ Guidelines for practice outside of class were reported in 2 of 3 studies that required and monitored home practice. Instructor qualifications were mentioned in 1 of 20 studies.

Eight of 11 RCTs used active control groups for comparison, including group Western exercise, ${ }^{29}$ stretching and toning, ${ }^{39}$ and combination of resistance and non-resistance 
training..$^{38}$ Five studies utilized educational programs..$^{9,23,32,34,37}$ Three studies employed a non-intervention control. ${ }^{26,27,33}$

\section{Outcomes measured}

Outcomes related to cognitive function were broadly categorized into the following cognitive domains: 1) global cognition: Mini Mental Status Exam (MMSE), Alzheimer's Disease Assessment Scale-Cognitive Subscale (ADAS-cog); 2) executive functions: measures of processing speed, attention, and working memory: Trail Making Test (TMT A\& B), digit span, digit symbol coding, spatial span forward/backward, Stroop color and word test, rule shift cards test, controlled oral word association test (COWAT), WAIS-R similarities, color trails test (CTT) trail 1-2; 3) language: verbal fluency (category/ semantic), Boston Naming Test (BNT); 4) learning and memory: list learning tasks: California Verbal Learning Test II (CVLT), Hopkins Verbal Learning Test (HVLT), Hong Kong List Learning Test (HKLLT), Rivermead Behavioral Memory Test (RBMT); and 5) spatial and quantitative: Rey-Osterrieth complex figure, clock drawing test (CDT), math word problems and calculations. Additional outcomes included neurobiological markers such as P300 amplitude and latency, MRI for whole brain volume, and psychiatric functioning (Neuropsychiatric Inventory; NPI). The three most widely used outcomes across studies were MMSE, TMT, and digit span test.

\section{Methodological quality of randomized clinical trials and bias risk}

Quality of reporting of clinical trial features was fair, although this varied considerably across the 11 RCTs (Table 3 ). Quality scores ranged from 4 to 11 (maximum score $=12$ ), with an average of 7.1. No studies were deemed fatally flawed, and 8 of the 11 studies had a score $\geq 6$ indicating a low risk of bias. . $^{9}, 23,26,29,33,34,37,39$

\section{Synthesis of studies of cognitively healthy adults}

Of the 11 studies that evaluated older adults without reported cognitive impairment, 4 were RCTs (total $n=421$ ) (Table 1). These RCTs all reported positive effects of Tai Chi on cognitive function including at least one measure of executive function. ${ }^{23,26,27,29}$ Metaanalyses including these four studies comparing Tai Chi to non-intervention controls for outcomes related to executive function indicated a large effect size, but high heterogeneity (Hedge's g=0.904; $\mathrm{p}=0.043, \mathrm{I}^{2}=92.02 \%$; Figure 2a). After excluding the one study with a low quality score indicating bias, ${ }^{27}$ the effect size was reduced but remained statistically significant and indicated no heterogeneity between studies (Hedge's $g=0.394 ; \mathrm{p}=0.004$ $\mathrm{I}^{2}=0 \%$ ). Meta-analyses of the two studies ${ }^{26,29}$ that compared Tai Chi to another exercise intervention (walking and "western exercise") also showed a modest positive effect size (Hedge's $\mathrm{g}=0.509 ; \mathrm{p}=0.003, \mathrm{I}^{2}=0 \%$; Figure $2 \mathrm{~b}$ ). Of note, one of these studies including a long-term follow-up reported sustained Tai Chi-related improvements in executive function at 1 year (i.e., 8 months post intervention). ${ }^{29}$

In addition, measures of learning and memory and semantic fluency (AVLT, CVLT,

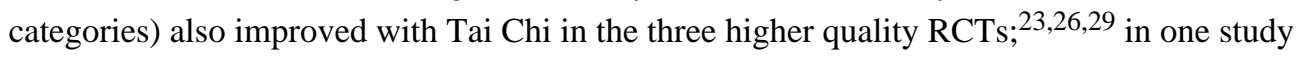
these improvements were greater relative to aerobic exercise. ${ }^{26}$ One study also reported 
improvements in overall global cognitive function (Mattis DRS) and total brain volume assessed via MRI. ${ }^{26}$

Findings from 6 of the 7 non-randomized and cross-sectional studies support an effect on cognitive function in healthy adults (Table 1). ${ }^{21,22,24,25,28,30}$ One small PNCS reported that 10 weeks of Tai Chi improved scores on two measures of executive function. ${ }^{25}$ Another small non-controlled study reported acutely enhanced performance in math computations. ${ }^{22}$ One CSS reported older adults who regularly trained with Tai Chi had better executive function/attention, learning and memory compared with matched sedentary adults or with conventional exercise. ${ }^{24} \mathrm{~A}$ second CSS reported that adults who practiced mind-body exercise (including Tai Chi) or cardiovascular exercise demonstrated similar levels of memory function. Exercise was better than non-exercisers, and those who practiced both mind-body and aerobic exercise cognitively outperformed all other groups. ${ }^{20}$

\section{Synthesis of studies of cognitively impaired adults}

Of the 9 studies that evaluated adults with some form of cognitive impairment, 7 were RCTs $(\text { total } n=843)^{9,32-35,37,38}$ (Table 2). All but one ${ }^{38}$ of these studies employed MMSE as a measure of global cognitive function. Meta-analyses of the 4 studies that included a nonintervention comparison ${ }^{9,32,33,37}$ indicated a small but statistically significant effect for Tai Chi on MMSE (Hedge's g=0.346; $\mathrm{p}=0.004, \mathrm{I}^{2}=0 \%$; Figure $2 \mathrm{c}$ ). When meta-analyses were limited to the 3 studies with quality scores $\geq 6,9,33,37$ effect sizes remained significant (Hedge's $\mathrm{g}=0.304 ; \mathrm{p}=0.033, \mathrm{I}^{2}=0 \%$ ). Meta-analyses including the 4 studies that compared Tai Chi to another active intervention (western exercise, cognitive behavioral therapy, Mahjong) ${ }^{32-34,39}$ showed a small positive significant effect of Tai Chi on MMSE (Hedge's $\mathrm{g}=0.300 ; \mathrm{p}=0.002, \mathrm{I}^{2}=0 \%$; Figure $2 \mathrm{~d}$ ). This effect remained when the one lower quality study $^{32}$ was removed (Hedge's $g=0.268 ; \mathrm{p}=0.010, \mathrm{I}^{2}=0 \%$ ).

Other outcomes observed in individual RCTs of cognitively impaired populations include a marked reduction in rates of progression to dementia $(4.3 \%$ vs. $16.6 \%$ in Tai Chi vs. western exercise) in one trial ${ }^{35}$ and improved executive function in Tai Chi compared with an arts and crafts intervention. ${ }^{32}$ One larger CSS $(n=782)$ reported the proportion of adults without cognitive impairment was highest in those with regular mind-body practice (including Tai Chi). In addition, participants who regularly practiced aerobic or mind-body exercises for $>5$ years had higher scores on MMSE, 10 min delayed recall, visual backward span, and CVFT $(\mathrm{p}<0.05){ }^{36}$

\section{Reports of safety and adverse events}

Six of the 11 RCTs reported on adverse events (AEs), however no RCTs described a formal protocol for systematically monitoring AEs. Two studies stated that no AEs were reported in either Tai Chi or comparison group. Four studies reported AE's including falls, hospitalization, side-effects of medication, and death; however, none were attributable to Tai Chi. 


\section{DISCUSSION}

The literature on non-pharmacological behavioral approaches to improve cognitive function in older adults has been growing. Tai Chi shows promise as an alternative multi-modal exercise for attenuating age-related cognitive decline and warrants further research. Our findings add to a growing body of studies evaluating a variety of other mind-body interventions (e.g., yoga, meditation, qigong) on cognitive function and provide further support for the effectiveness of multi-modal behavioral interventions. ${ }^{13,40}$

\section{Tai Chi for cognitively intact adults}

Results of our meta-analyses support small to moderate, but clinically relevant improvements in executive function following 10 weeks to 1 year of Tai Chi training in cognitively intact adults. Effect sizes were equivalent to those reported following other exercise ${ }^{3,4}$ and cognitive training. ${ }^{41}$ Benefits were apparent in RCTs that compared Tai Chi to other exercise interventions suggesting that Tai Chi's effects are not simply due to group social support or placebogenic expectation for improvement. While the positive effects of Tai Chi on executive function persisted when our analyses were limited to studies of higher methodological quality, more definitive conclusions regarding Tai Chi's potential benefits will require results from a greater number of larger, well-designed studies.

Executive function is an umbrella term for cognitive processes that regulate, control, and manage other cognitive processes such as planning, working memory, attention, problem solving, verbal reasoning, inhibition, mental flexibility, task switching, and initiation and monitoring of actions. ${ }^{42}$ Executive function is increasingly appreciated as a key component of healthy balance and postural control. ${ }^{43}$ Improvement in this domain of cognitive function may thus play a role in mediating Tai Chi's effects on balance and motor control. The link between Tai Chi-associated increases in executive function and motor control is supported by some recent studies. ${ }^{25,44}$

Evidence for the impact of Tai Chi on other domains of cognitive function in cognitively intact adults is less conclusive. Individual studies reported improvements in outcomes related to learning, memory, language, global cognitive function, as well as total brain volume assessed with MRI. These outcomes, however, were not employed consistently across studies precluding quantitative synthesis.

\section{Tai Chi for cognitively impaired}

Tai Chi may benefit cognitive function in cognitively impaired adults. Results of our metaanalyses support small to moderate, but clinically relevant improvements in global cognitive function (MMSE) following 12 weeks to 1 year of Tai Chi training, equivalent to effects reported following other exercise ${ }^{3,4}$ and cognitive training. ${ }^{41}$ As in studies of cognitively intact adults, these benefits were apparent in RCTs that compared Tai Chi to other exercise interventions, as well as non-active interventions, and persisted when methodologically biased studies were excluded from analyses. Evidence for the impact of Tai Chi on specific domains of cognitive function in cognitively impaired adults is less conclusive. 


\section{Safety}

Poor reporting of protocols for systematically monitoring adverse events limits the conclusions that can be drawn regarding the safety of Tai Chi in populations with and without cognitive impairment. However, in the subset of studies that did report adverse events, there were no reports that could be directly attributed to Tai Chi. These observations support other study findings - including populations with serious balance impairments, cardiovascular risk factors, and affective disorders -- that Tai Chi can be safely administered with few serious adverse events. ${ }^{14-16}$ Of note, all studies in this review relied on simplified forms, thereby minimizing potential psychological distress associated with memorizing complex routines. For populations with existing cognitive impairment, interventions based on long and complex choreographies should be contraindicated.

\section{How might Tai Chi positively impact cognitive function?}

It has been hypothesized that there are at least six potentially therapeutic elements inherent in Tai Chi training that may underlie its impact on cognitive function. ${ }^{10}$ First, Tai Chi is a moderately aerobic exercise, with metabolic equivalents estimated between 1.5 and 4.0. This aerobic intensity overlaps with brisk walking which has been shown to have positive effects on cognitive function. ${ }^{45}$ Importantly, the impact of exercise is supported by studies associating functional changes with neurophysiological changes such as increased brain derived neurotrophic factor and plasticity in brain morphology and function, including processes central to executive function. ${ }^{5,46-48}$ Future studies might evaluate how Tai Chi vs. unimodal exercise programs (e.g., stationary cycling) compare with respect to their impact on brain plasticity and activation of neural networks during functional tasks. Second, Tai Chi trains agility and mobility, which some evidence suggests impacts cognitive function via neurophysiological pathways unique to those associated with aerobic exercise. One study reported that aerobic and muscular fitness were both associated with executive function, where as motor fitness (agility and mobility) was associated with both executive control and perceptual speed tasks. Paralleling these functional changes were distinct patterns of activation assessed using fMRI during the Flanker Task. ${ }^{11}$ Future studies might use functional imaging paradigms to explore how biomechanically repetitive exercises that target aerobic and muscle fitness (e.g., treadmill walking) compare with exercises like Tai Chi that also train agility and dynamic postural control. Third, Tai Chi involves learning and memorization of new skills and movement patterns; studies of other skill-based learning activities including dance, juggling, and music have shown improvements in cognitive function and underlying neural mechanisms. ${ }^{49,50}$ For example, learning juggling has been associated with increased gray matter in the occipito-temporal cortex ${ }^{51}$ and dance training with hippocampal formation. ${ }^{52}$ Better understanding how the learning of Tai Chi routines that vary in choreographical complexity impacts brain structure and function will greatly enhance our mechanistic understanding of Tai Chi on cognitive function. Fourth, Tai Chi includes training in sustained attentional focus, shifting, and multi-tasking which could help train working memory, divided attention, cognitive flexibility, and overall executive function. One study that employed a computerized visual and finger-pointing paradigm reported that compared to age-matched Tai Chi naïve controls, older Tai Chi practitioners have improved hand-eye coordination and movement times during tasks involving cognitive processing. ${ }^{44}$ Fifth, the meditative and relaxation training of Tai Chi have been shown to 
reduce anxiety and depression, ${ }^{14}$ which may impact cortisol and other stress-related pathways of cognitive decline. ${ }^{7}$ The relevance of this component of Tai Chi is supported by studies linking meditation training with improvements in multiple functional and neurophysiological aspects of cognitive performance. ${ }^{12}$ Finally, greater time allocated to leisure activities and social support has been associated with improved or preserved cognitive function. ${ }^{8}$ In summary, these six interdependent therapeutic elements of Tai Chi may afford greater effectiveness in enhancing cognitive function, compared to unimodal interventions that target a more limited set of mechanisms and physiological pathways.

\section{Limitations and Suggestions for Future Research}

There are a number of limitations to this study. First, our analysis was limited to studies in the English language. Including studies in other languages may have better represented the evidence to date and improved generalizability to other cultures. Second, due to heterogeneity in outcomes employed in trials, we necessarily limited our meta-analyses to only the most commonly measured outcomes. Until more studies are available with common outcomes reflecting other domains, it will be difficult to more broadly evaluate Tai Chi and cognitive function. Some studies with poorer methodological quality were included in this review. In addition to better reporting of key design features (e.g., blinding, randomization, adverse event reporting), future studies should report features specifically relevant to Tai Chi studies (e.g., details, rationale and validity of training protocols). This will enable future reviews to better evaluate protocol-specific effects. Third, our pooling of studies into two broad groups - cognitively healthy and cognitively impaired population - does not capture the diversity and complexity of participants across studies, and thus limits the inferences we can draw regarding the benefits of Tai Chi to specific populations. Future reviews should further stratify results based on more fine-grained classification of levels of cognitive impairment as well as cognition-related comorbidities. Finally, given the unique multicomponent nature of Tai Chi, outcomes characterizing complex physiological (e.g. aerobic capacity, neuroendocrine function, motor control) and behavioral measures (e.g. stress, mood, body awareness, perceived social support) should be evaluated and explored as mechanisms or mediators of Tai Chi's effects.

\section{CONCLUSIONS}

Our meta-analysis and broader systematic review suggest that Tai Chi may offer a safe, nonpharmacological approach to enhancing cognitive function in older adults. Larger, more robust trials with longer follow-up periods and standardized neuropsychological outcome measures are needed before more definitive conclusions can be drawn. If future research supports the benefits of Tai Chi for cognitive function, this would add to the literature supporting other Tai Chi-associated benefits for older adults, including positive effects on balance and fall prevention, cardiovascular risk, musculoskeletal pain, immune function and psychological well-being. ${ }^{10}$ Tai Chi may be an attractive first line intervention for adults interested in integrated long-term strategies for healthy aging. 


\section{Acknowledgments}

Funding Sources: This publication was made possible by grant number R21 AT005501-01A1 from the National Center for Complementary and Alternative Medicine (NCCAM) at the National Institutes of Health (NIH). Its contents are solely the responsibility of the authors and do not necessarily represent the official views of the NCCAM or the NIH.

Sponsor's Role: None

\section{REFERENCES}

1. Plassman BL, Langa KM, Fisher GG, et al. Prevalence of dementia in the United States: The aging, demographics, and memory study. Neuroepidemiology. 2007; 29:125-132. [PubMed: 17975326]

2. Wimo A, Winblad B, Jonsson L. The worldwide societal costs of dementia: Estimates for 2009. Alzheimers Dementia. 2010; 6:98-103.

3. Angevaren M, Aufdemkampe G, Verhaar HJ, et al. Physical activity and enhanced fitness to improve cognitive function in older people without known cognitive impairment. Cochrane Database Syst Rev. 2008 CD005381.

4. Hamer M, Chida Y. Physical activity and risk of neurodegenerative disease: A systematic review of prospective evidence. Psychol Med. 2009; 39:3-11. [PubMed: 18570697]

5. Fang ZH, Lee CH, Seo MK, et al. Effect of treadmill exercise on the BDNF-mediated pathway in the hippocampus of stressed rats. Neurosci Res. 2013

6. Lopez-Lopez C, LeRoith D, Torres-Aleman I. Insulin-like growth factor I is required for vessel remodeling in the adult brain. Proc Natl Acad Sci U S A. 2004; 101:9833-9838. [PubMed: 15210967]

7. Lupien SJ, de Leon M, de Santi S, et al. Cortisol levels during human aging predict hippocampal atrophy and memory deficits. Nat Neurosci. 1998; 1:69-73. [PubMed: 10195112]

8. Verghese J, Lipton RB, Katz MJ, et al. Leisure activities and the risk of dementia in the elderly. N Engl J Med. 2003; 348:2508-2516. [PubMed: 12815136]

9. Burgener SC, Yang Y, Gilbert R, et al. The effects of a multimodal intervention on outcomes of persons with early-stage dementia. Am J Alzheimers Dis Other Dement. 2008; 23:382-394.

10. Wayne PM, Fuerst ML. The Harvard Medical School Guide To Tai Chi. United States of America, Shambhala Publications Inc. 2013

11. Voelcker-Rehage C, Godde B, Staudinger UM. Cardiovascular and coordination training differentially improve cognitive performance and neural processing in older adults. Front Hum Neurosci. 2011; 5:26. [PubMed: 21441997]

12. Chiesa A, Calati R, Serretti A. Does mindfulness training improve cognitive abilities? A systematic review of neuropsychological findings. Clin Psychol Rev. 2011; 31:449-464. [PubMed: 21183265]

13. Prakash R, Rastogi P, Dubey I, et al. Long-term concentrative meditation and cognitive performance among older adults. Neuropsychol Develop Cogn B Aging Neuropsychol Cogn. 2012; 19:479-494.

14. Wang C, Bannuru R, Ramel J, et al. Tai Chi on psychological well-being: Systematic review and meta-analysis. BMC Complement Altern Med. 2010; 10:23. [PubMed: 20492638]

15. Yeh GY, Wang C, Wayne PM, et al. Tai chi exercise for patients with cardiovascular conditions and risk factors: A Systematic Review. J Cardiopulm Rehabil Prev. 2009; 29:152-160. [PubMed: 19471133]

16. Logghe IH, Verhagen AP, Rademaker AC, et al. The effects of Tai Chi on fall prevention, fear of falling and balance in older people: A meta-analysis. Prev Med. 2010; 51:222-227. [PubMed: 20558197]

17. Liberati A, Altman DG, Tetzlaff J, et al. The PRISMA statement for reporting systematic reviews and meta-analyses of studies that evaluate healthcare interventions: Explanation and elaboration. BMJ. 2009; 339:b2700. [PubMed: 19622552] 
18. Borenstein, M.; Hedges, LV.; Higgins, JPT., et al. West Sussex, UK: 2009. Introduction to MetaAnalysis.

19. Furlan AD, Pennick V, Bombardier C, et al. 2009 Updated Method Guidelines for Systematic Reviews in the Cochrane Back Review Group. Spine. 2009; 34:1929-1941. [PubMed: 19680101]

20. Chan AS, Ho YC, Cheung MC, et al. Association between mind-body and cardiovascular exercises and memory in older adults. J Am Geriatr Soc. 2005; 53:1754-1760. [PubMed: 16181176]

21. Coubard OA, Duretz S, Lefebvre V, et al. Practice of contemporary dance improves cognitive flexibility in aging. Front Aging Neurosci. 2011; 3:13. [PubMed: 21960971]

22. Field T, Diego M, Hernandez-Reif M. Tai chi/yoga effects on anxiety, heartrate, EEG and math computations. Complement Ther Clin Pract. 2010; 16:235-238. [PubMed: 20920810]

23. Lavretsky H, Alstein LL, Olmstead RE, et al. Complementary use of tai chi chih augments escitalopram treatment of geriatric depression: A randomized controlled trial. Am J Geriatr Psychiatry. 2011; 19:839-850. [PubMed: 21358389]

24. Man DW, Tsang WW, Hui-Chan CW. Do older t'ai chi practitioners have better attention and memory function? J Altern Complement Med. 2010; 16:1259-1264. [PubMed: 21138387]

25. Matthews MM, Williams HG. Can Tai chi enhance cognitive vitality? A preliminary study of cognitive executive control in older adults after A Tai chi intervention. JSC Med Assoc. 2008; 104:255-257.

26. Mortimer JA, Ding D, Borenstein AR, et al. Changes in brain volume and cognition in a randomized trial of exercise and social interaction in a community-based sample of non-demented Chinese elders. J Alzheimers Dis. 2012; 30:757-766. [PubMed: 22451320]

27. Nguyen MH, Kruse A. A randomized controlled trial of Tai chi for balance, sleep quality and cognitive performance in elderly Vietnamese. Clin Interven Aging. 2012; 7:185-190.

28. Reid-Arndt SA, Matsuda S, Cox CR. Tai Chi effects on neuropsychological, emotional, and physical functioning following cancer treatment: A pilot study. Complemen Ther Clin Pract. 2012; 18:26-30.

29. Taylor-Piliae RE, Newell KA, Cherin R, et al. Effects of Tai Chi and Western exercise on physical and cognitive functioning in healthy community-dwelling older adults. J Aging Phys Act. 2010; 18:261-279. [PubMed: 20651414]

30. Wu W-L. The effect of Chinese Yuanji-Dance on dynamic balance and the associated attentional demands in elderly adults. J Sports Sci Med. 2010; 9:119-126. [PubMed: 24149395]

31. Chang JY, Tsai PF, Beck C, et al. The effect of tai chi on cognition in elders with cognitive impairment. Med Surg Nurs. 2011; 20:63-69.

32. Cheng ST, Chow PK, Song YQ, et al. Mental and physical activities delay cognitive decline in older persons with dementia. Am J Geriatr Psychiatry. 2012

33. Dechamps A, Diolez P, Thiaudiere E, et al. Effects of exercise programs to prevent decline in health-related quality of life in highly deconditioned institutionalized elderly persons: a randomized controlled trial. Arch Intern Med. 2010; 170:162-169. [PubMed: 20101011]

34. Deschamps A, Onifade C, Decamps A, et al. Health-related quality of life in frail institutionalized elderly: Effects of a cognition-action intervention and Tai Chi. J Aging Phys Act. 2009; 17:236248. [PubMed: 19451671]

35. Lam LC, Chau RC, Wong BM, et al. Interim follow-up of a randomized controlled trial comparing Chinese style mind body (Tai Chi) and stretching exercises on cognitive function in subjects at risk of progressive cognitive decline. Int J Geriatr Psychiatry. 2011; 26:733-740. [PubMed: 21495078]

36. Lam LC, Tam CW, Lui VW, et al. Modality of physical exercise and cognitive function in Hong Kong older Chinese community. Int J Geriatr Psychiatry. 2009; 24:48-53. [PubMed: 18615844]

37. Tsai PF, Chang JY, Beck C, et al. A Pilot Cluster-Randomized Trial of a 20-Week Tai Chi Program in Elders With Cognitive Impairment and Osteoarthritic Knee: Effects on pain and other health outcomes. J Pain Symp Manage. 2013; 45:660-669.

38. Wang W, Sawada M, Noriyama Y, et al. Tai Chi exercise versus rehabilitation for the elderly with cerebral vascular disorder: A single-blinded randomized controlled trial. Psychogeriatrics. 2010; 10:160-166. [PubMed: 20860572] 
39. Lam LC, Chau RC, Wong BM, et al. A 1-year randomized controlled trial comparing mind body exercise (Tai Chi) with stretching and toning exercise on cognitive function in older Chinese adults at risk of cognitive decline. J Am Med Dir Assoc. 2012; 13:568, e515-e520. [PubMed: 22579072]

40. Oh B, Butow PN, Mullan BA, et al. Effect of medical Qigong on cognitive function, quality of life, and a biomarker of inflammation in cancer patients: A randomized controlled trial. Support Care Cancer. 2012; 20:1235-1242. [PubMed: 21688163]

41. Valenzuela M, Sachdev P. Can cognitive exercise prevent the onset of dementia? Systematic review of randomized clinical trials with longitudinal follow-up. Am J Geriatr Psychiatry. 2009; 17:179-187. [PubMed: 19225276]

42. Chan RC, Shum D, Toulopoulou T, et al. Assessment of executive functions: Review of instruments and identification of critical issues. Arch Clin Neuropsychol. 2008; 23:201-216. [PubMed: 18096360]

43. Hausdorff JM, Buchman AS. What links gait speed and MCI with dementia? A fresh look at the association between motor and cognitive function. J Gerontol A Biol Sci Med Sci. 2013; 68:409411. [PubMed: 23401565]

44. Tsang WW, Kwok JC, Hui-Chan CW. Effects of aging and tai chi on a finger-pointing task with a choice paradigm. Evidence-based Complementary and Alternative Medicine : eCAM. 2013; 2013:653437. [PubMed: 23476699]

45. Erickson KI, Voss MW, Prakash RS, et al. Exercise training increases size of hippocampus and improves memory. Proc Natl Acad Sci U S A. 2011; 108:3017-3022. [PubMed: 21282661]

46. Colcombe SJ, Erickson KI, Scalf PE, et al. Aerobic exercise training increases brain volume in aging humans. J Gerontol A Biol Sci Med Sci. 2006; 61:1166-1170. [PubMed: 17167157]

47. Pereira AC, Huddleston DE, Brickman AM, et al. An in vivo correlate of exercise-induced neurogenesis in the adult dentate gyrus. Proc Natl Acad Sci U S A. 2007; 104:5638-5643. [PubMed: 17374720]

48. Voss MW, Prakash RS, Erickson KI, et al. Plasticity of brain networks in a randomized intervention trial of exercise training in older adults. Front Aging Neurosci. 2010; 2:32. [PubMed: 20890449]

49. Herdener M, Esposito F, di Salle F, et al. Musical training induces functional plasticity in human hippocampus. J Neurosci. 2010; 30:1377-1384. [PubMed: 20107063]

50. Kattenstroth JC, Kolankowska I, Kalisch T, et al. Superior sensory, motor, and cognitive performance in elderly individuals with multi-year dancing activities. Front Aging Neurosci. 2010; 2:31. [PubMed: 20725636]

51. Driemeyer J, Boyke J, Gaser C, et al. Changes in gray matter induced by learning--revisited. PloS One. 2008; 3:e2669. [PubMed: 18648501]

52. Hufner K, Binetti C, Hamilton DA, et al. Structural and functional plasticity of the hippocampal formation in professional dancers and slackliners. Hippocampus. 2011; 21:855-865. [PubMed: 20572197] 
Identification

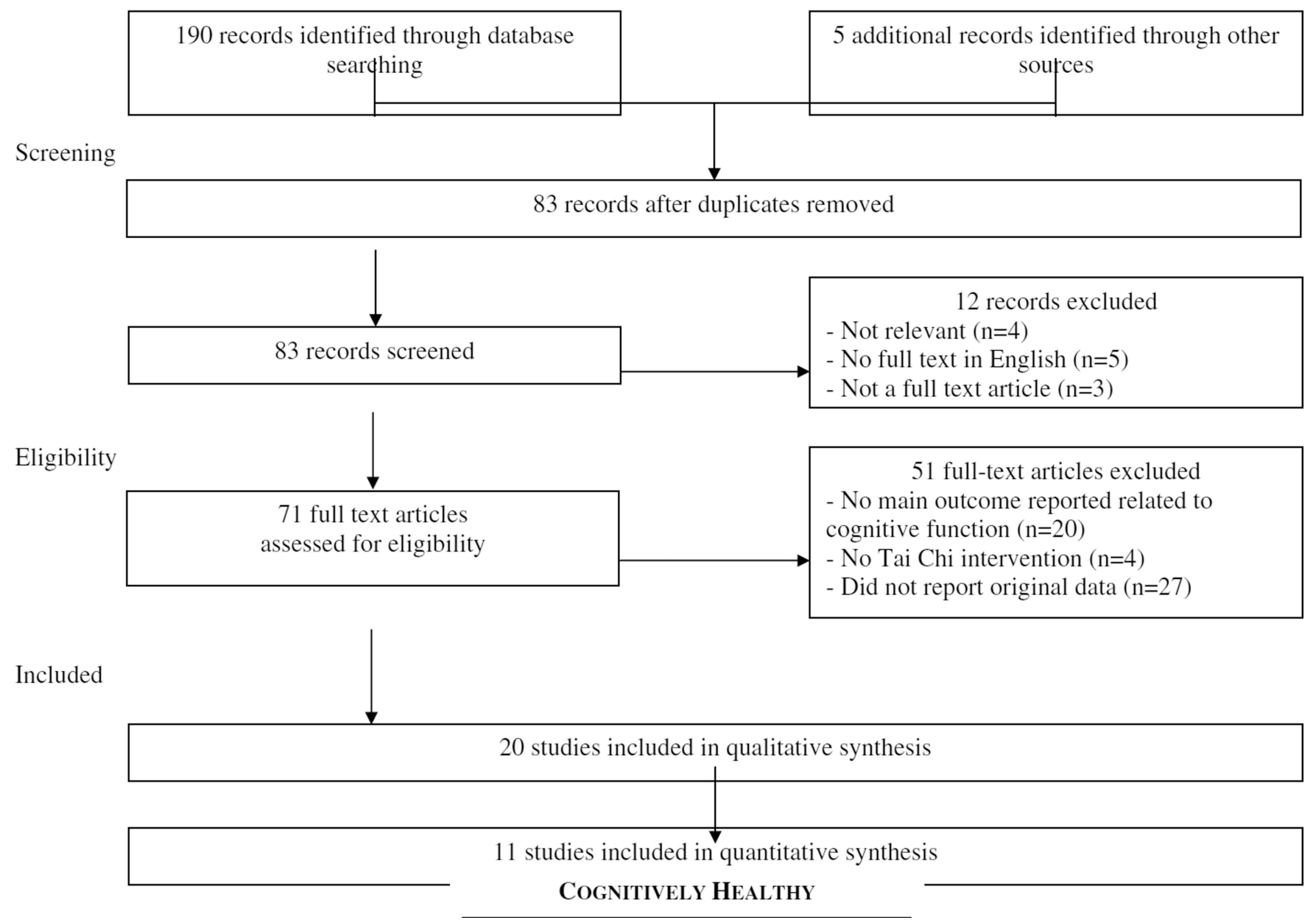

Figure 1.

Summary of the flow of our literature search according to the Preferred Reporting Items for Systematic Reviews and Meta-Analyses (PRISMA) guidelines. 


\section{Executive Function: Tai Chi vs Controls}

Mbdel Studyname Comparison

Lavetsky, 2011

Mortimer, 2012

Nguyen, 2012

Fixed

Random

TMT-A
Combined
Combined
Combined

Statistics for each study Hedges's Lower Upper g limit limit $p$-Value $\begin{array}{llll}0.408 & -0.067 & 0.883 & 0.092\end{array}$ $\begin{array}{llll}0.529 & 0.017 & 1.041 & 0.043\end{array}$ $\begin{array}{llll}2.458 & 1.850 & 3.066 & 0.000\end{array}$ $\begin{array}{llll}0.296 & -0.120 & 0.711 & 0.163\end{array}$

$\begin{array}{llll}0.728 & 0.484 & 0.972 & 0.000\end{array}$ $\begin{array}{llll}0.904 & 0.026 & 1.781 & 0.043\end{array}$
Hedges's g and $95 \%$ C
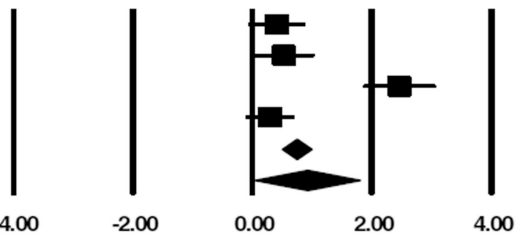

Fav ours Controls

a

\section{Executive Function: Tai Chi vs Active Comparisons}

Mbdel Studyname Comparison

Mortimer, 2012 Walking Combined

Taylor-Piliae, 2010 Westem Exercise Combined

Fixed

Random
Statistics for each study

$\begin{array}{cccc}\begin{array}{c}\text { Hedges's } \\ \mathbf{g}\end{array} & \begin{array}{c}\text { Lower } \\ \text { limit }\end{array} & \begin{array}{c}\text { Upper } \\ \text { limit }\end{array} & \text { p-Value } \\ 0.439 & -0.068 & 0.946 & 0.090 \\ 0.565 & 0.109 & 1.021 & 0.015 \\ 0.509 & 0.170 & 0.848 & 0.003 \\ 0.509 & 0.170 & 0.848 & 0.003\end{array}$

Hedges's g and $95 \% \mathrm{Cl}$
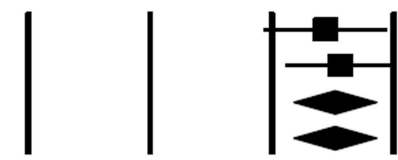

$-2.00$
0.00

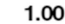

(a) 
COGNITIVELY IMPAIRED

\section{Global Cognition: Tai Chi vs Controls}

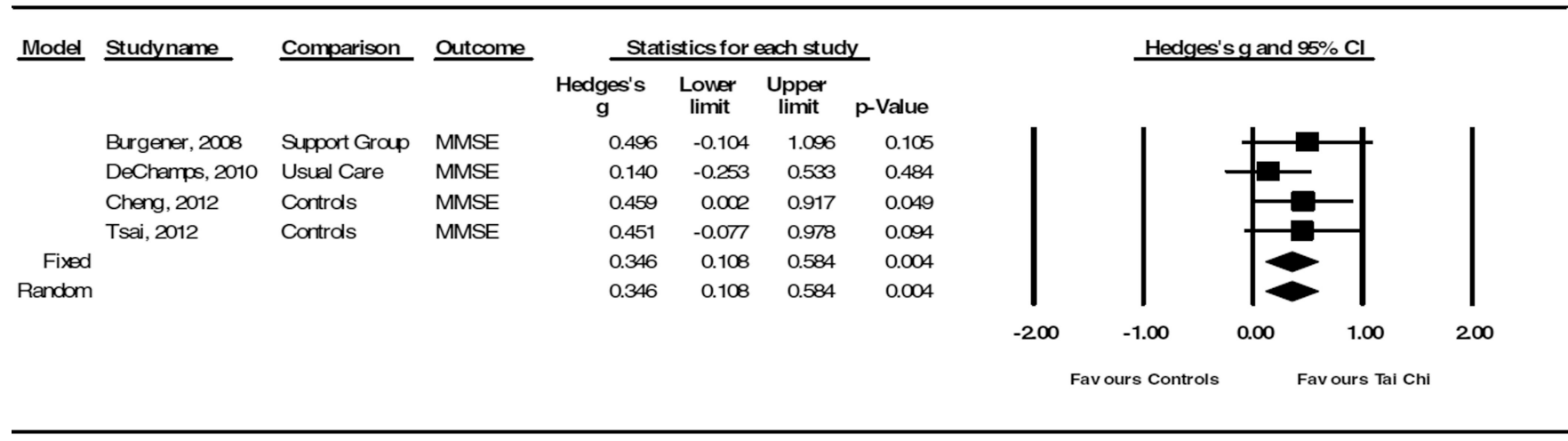

C

\section{Global Cognition: Tai Chi vs Active Comparisons}

\begin{tabular}{|c|c|c|c|c|c|c|c|}
\hline \multirow[t]{2}{*}{ Model } & \multirow[t]{2}{*}{ Study name } & \multirow[t]{2}{*}{ Comparison } & \multirow[t]{2}{*}{ Outcome } & \multicolumn{4}{|c|}{ Statistics for each study } \\
\hline & & & & $\begin{array}{l}\text { Hedges's } \\
\text { g }\end{array}$ & $\begin{array}{l}\text { Lower } \\
\text { limit }\end{array}$ & $\begin{array}{l}\text { Upper } \\
\text { limit }\end{array}$ & $\mathrm{p}$-Value \\
\hline & DeChamps, 2009 & Cognition-Action Ex & MSE & 0.015 & -0.633 & 0.663 & 0.963 \\
\hline & DeChamps, 2010 & Cognition-Action Ex & MSE & 0.145 & -0.261 & 0.551 & 0.485 \\
\hline & Cheng, 2012 & Mahjong & MMSE & 0.456 & 0.002 & 0.910 & 0.049 \\
\hline & Lam, 2012 & Stretch/Toning Ex & MSE & 0.356 & 0.101 & 0.611 & 0.006 \\
\hline Fixed & & & & 0.300 & 0.113 & 0.486 & 0.002 \\
\hline Random & & & & 0.300 & 0.113 & 0.486 & 0.002 \\
\hline
\end{tabular}

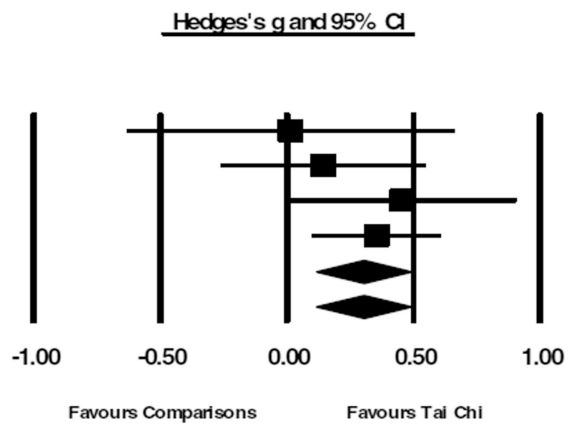

d

Figure 2.

Results of meta-analyses evaluating Tai Chi for cognitive function. $2 \mathrm{a}$ and $2 \mathrm{~b}$ summarize results for comparisons between Tai Chi and non-active and active controls, respectfully, for executive function in adults without existing cognitive impairment. $2 \mathrm{c}$ and $2 \mathrm{~d}$ summarize results for comparisons between Tai Chi and non-active and active controls, respectfully, for global cognition in cognitively impaired adults. 

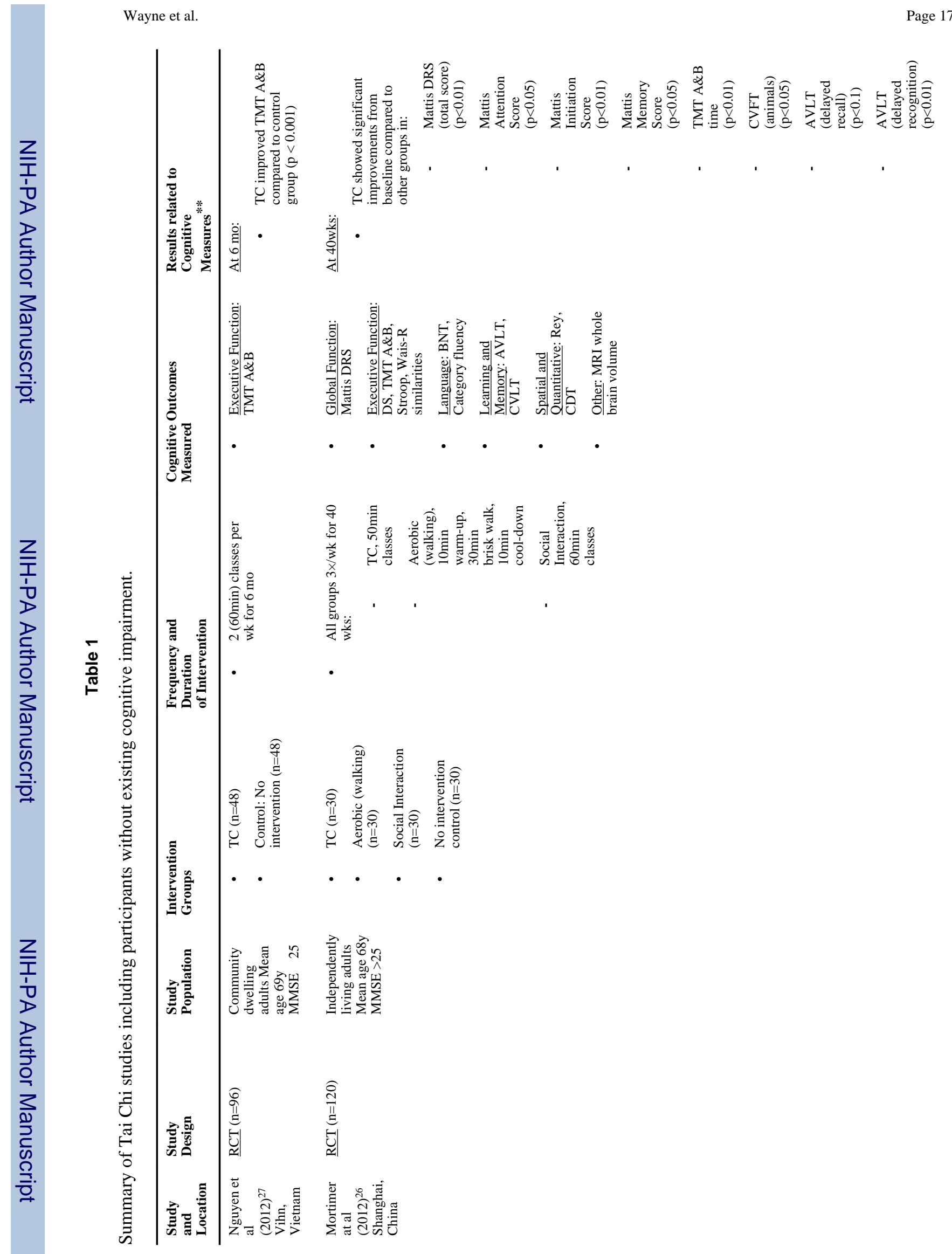

$J$ Am Geriatr Soc. Author manuscript; available in PMC 2015 January 02. 


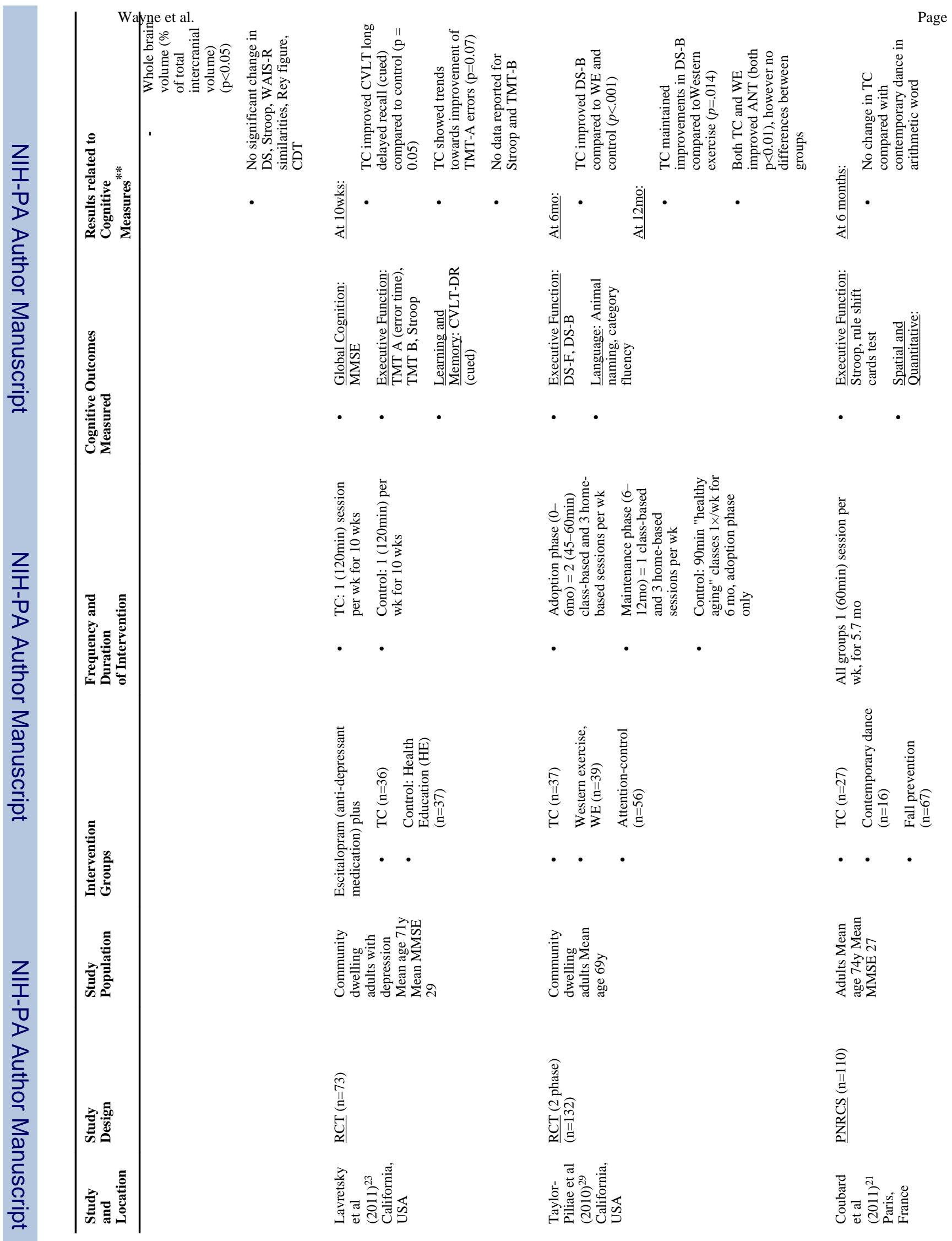

$J$ Am Geriatr Soc. Author manuscript; available in PMC 2015 January 02. 


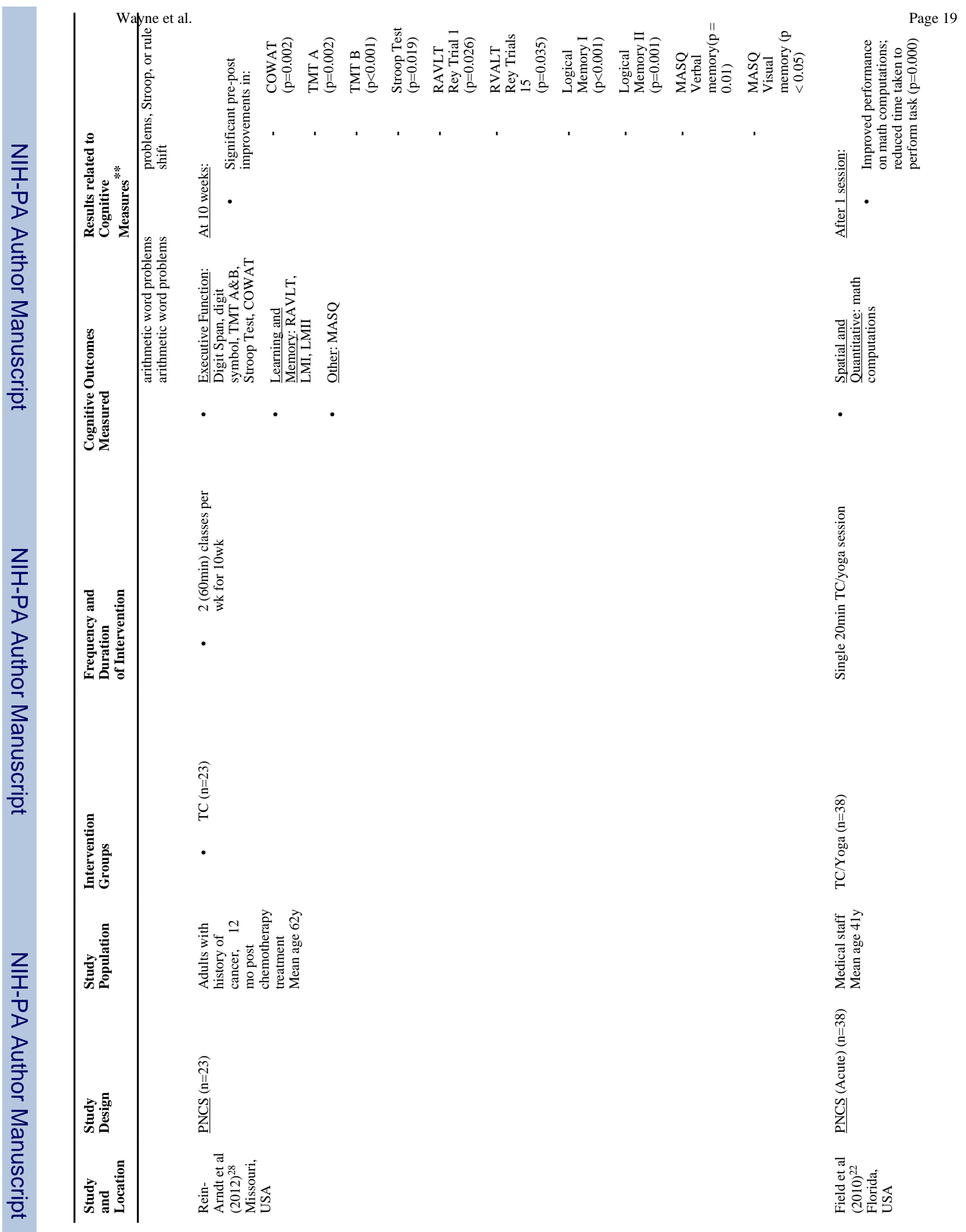

$J$ Am Geriatr Soc. Author manuscript; available in PMC 2015 January 02. 


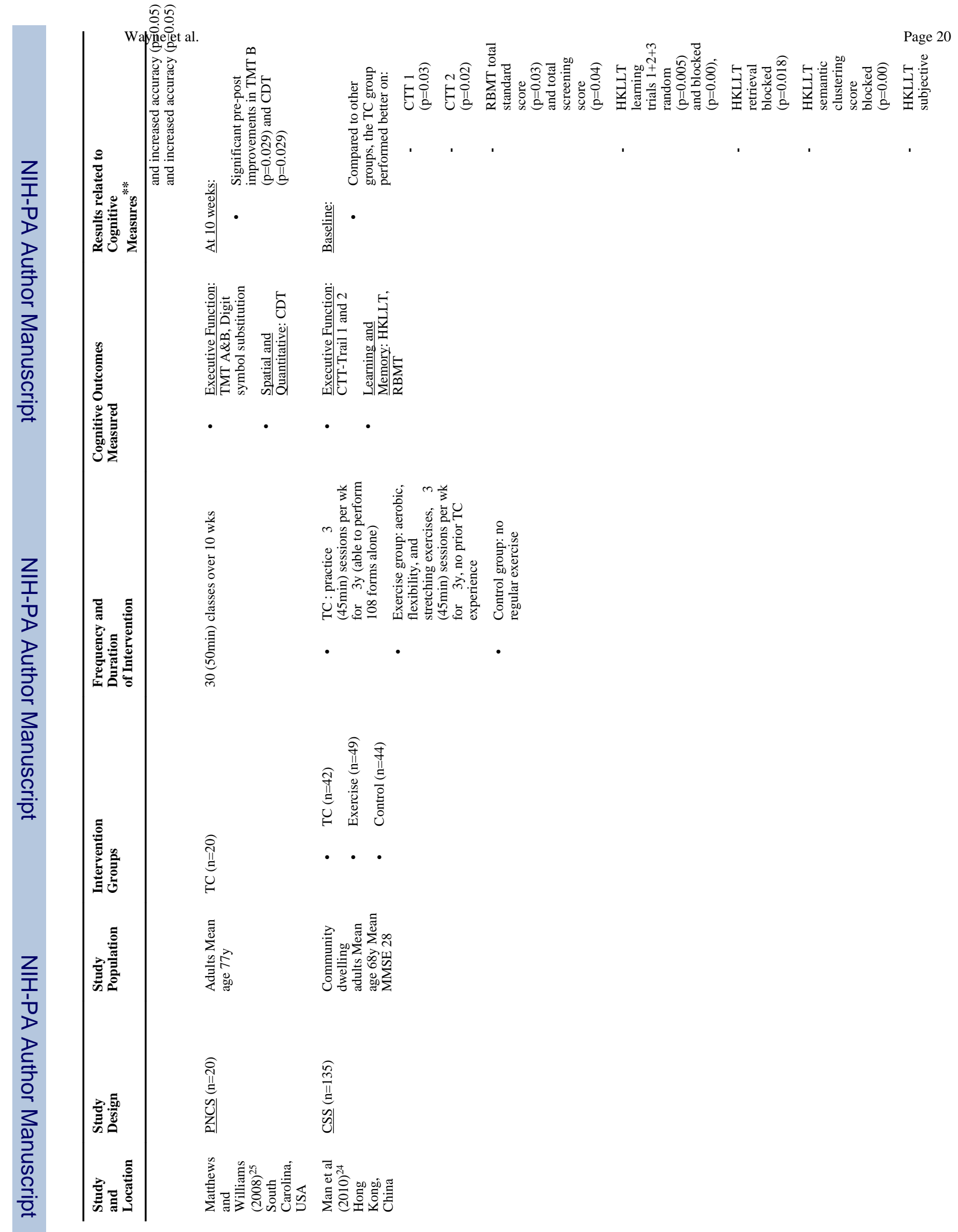

$J$ Am Geriatr Soc. Author manuscript; available in PMC 2015 January 02. 

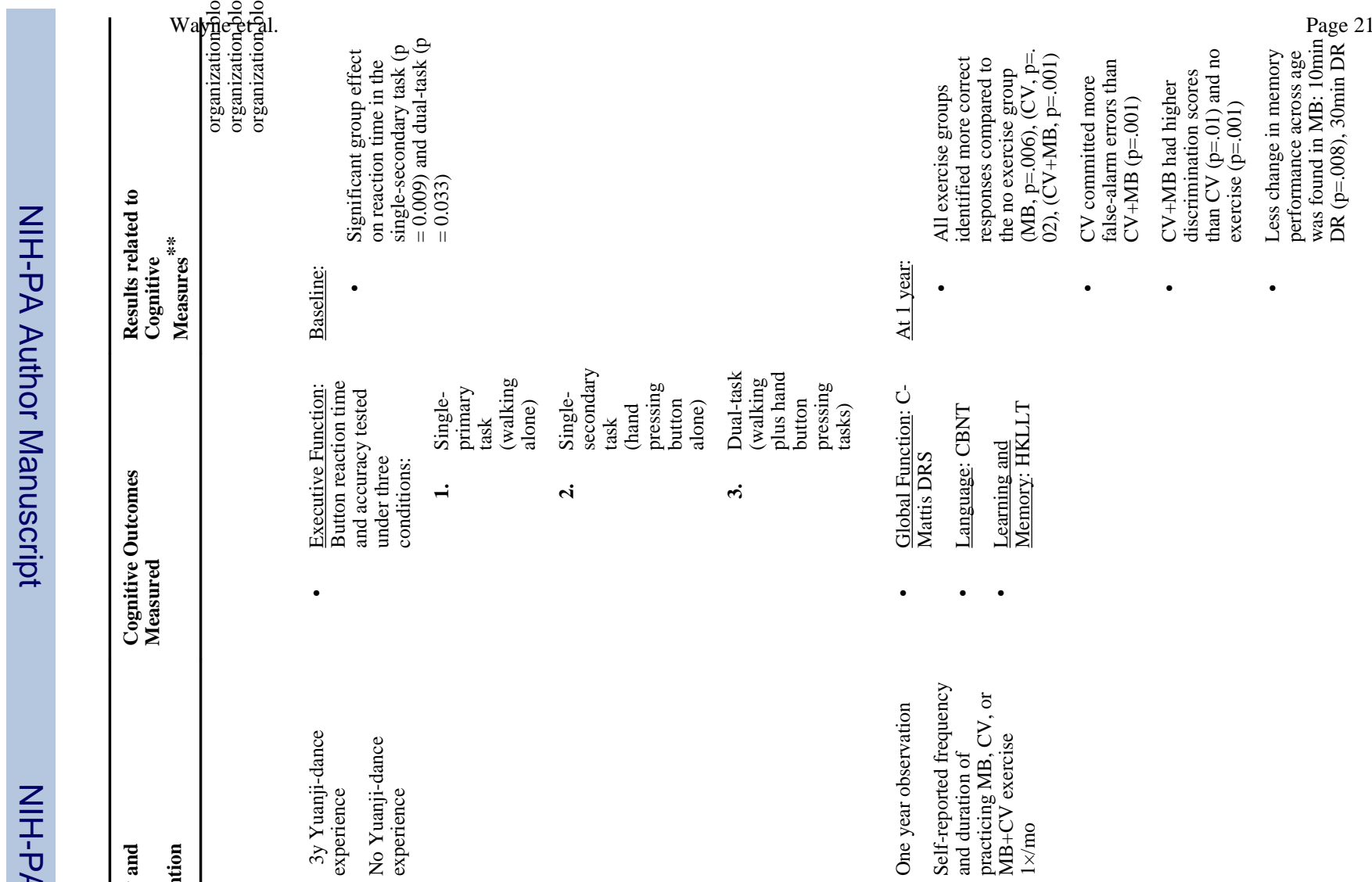

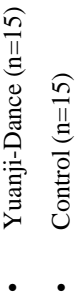

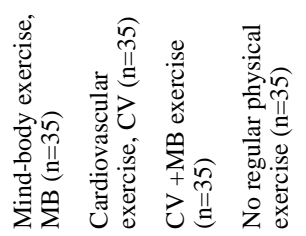

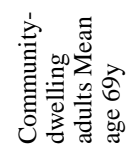

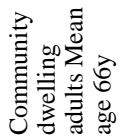

ָึ

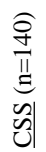

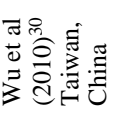

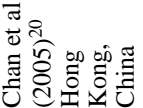

$J$ Am Geriatr Soc. Author manuscript; available in PMC 2015 January 02. 


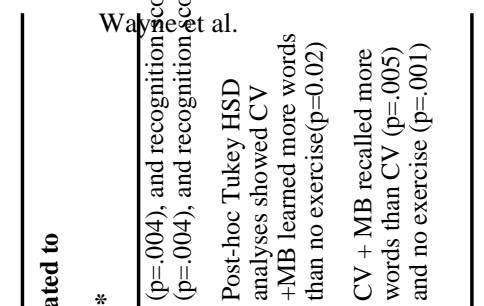

Page 22

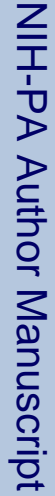

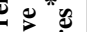




.

.




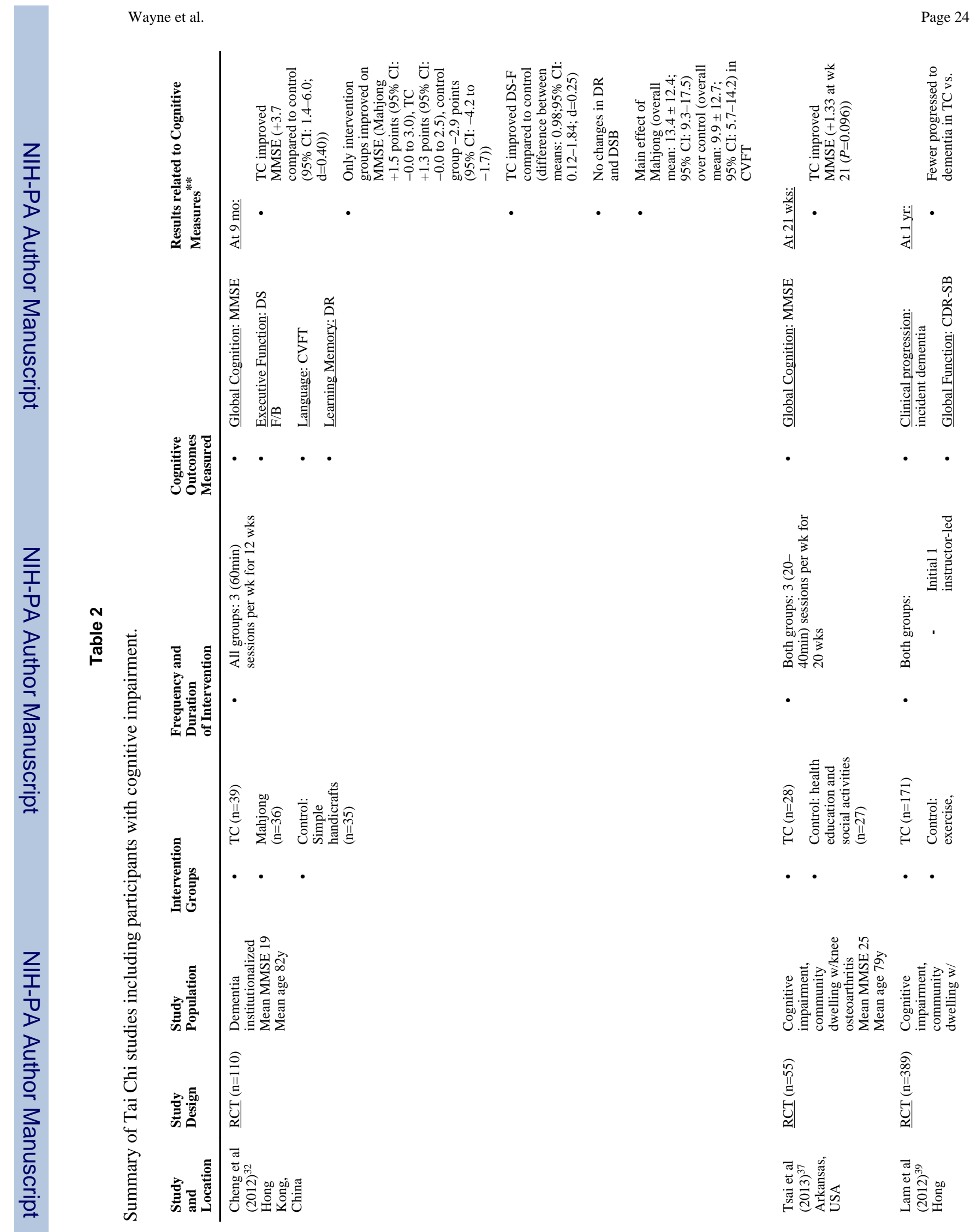

$J$ Am Geriatr Soc. Author manuscript; available in PMC 2015 January 02. 


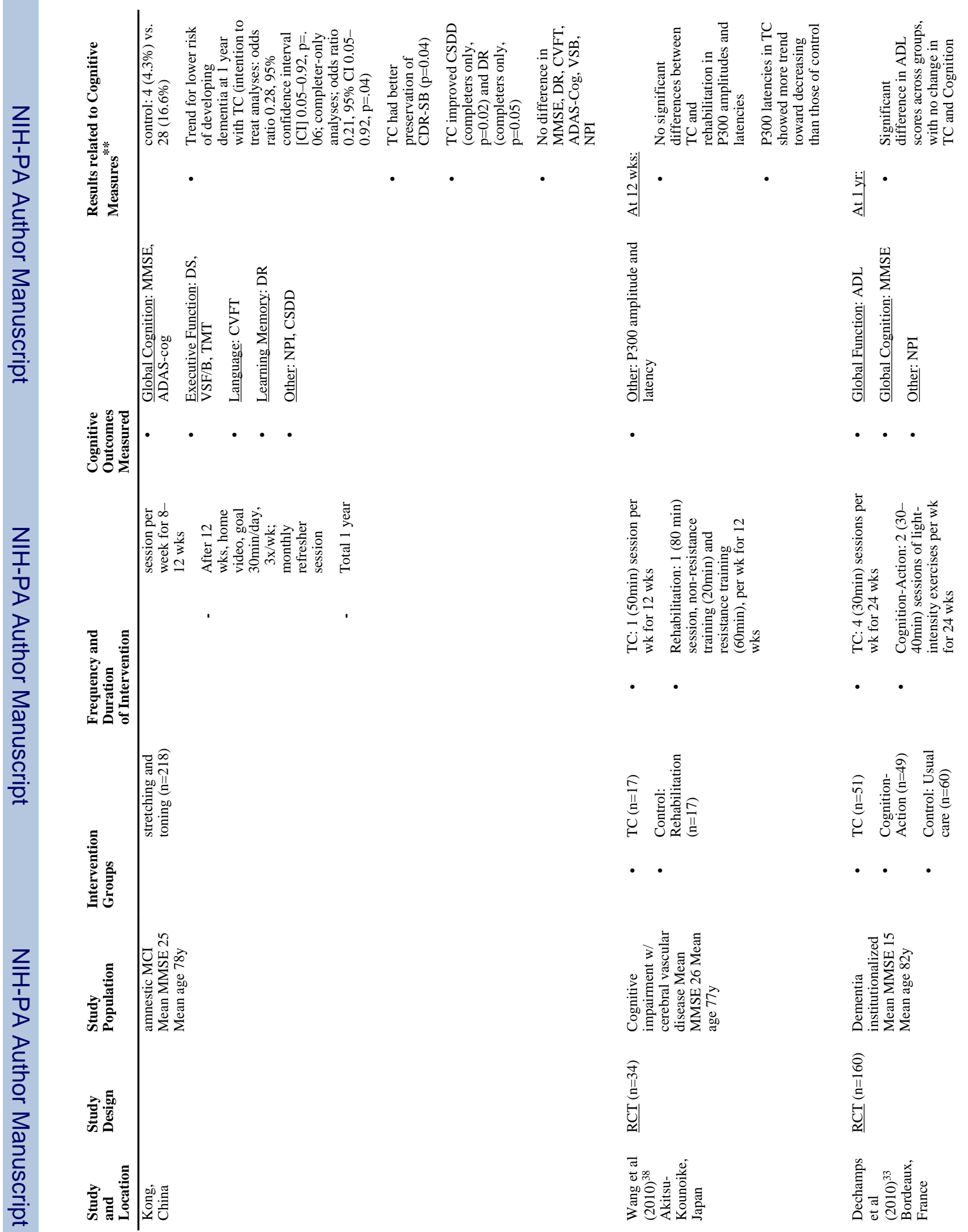




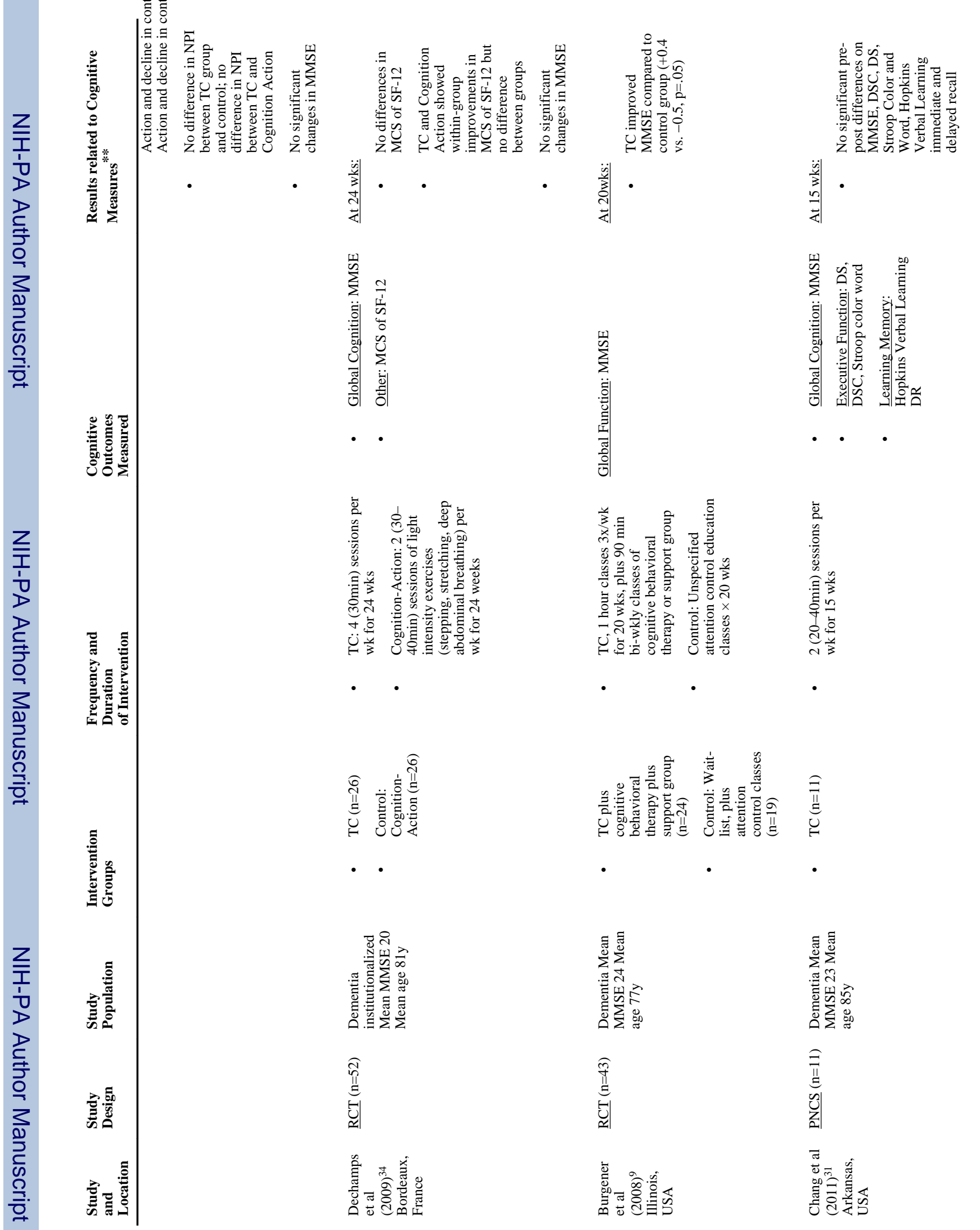




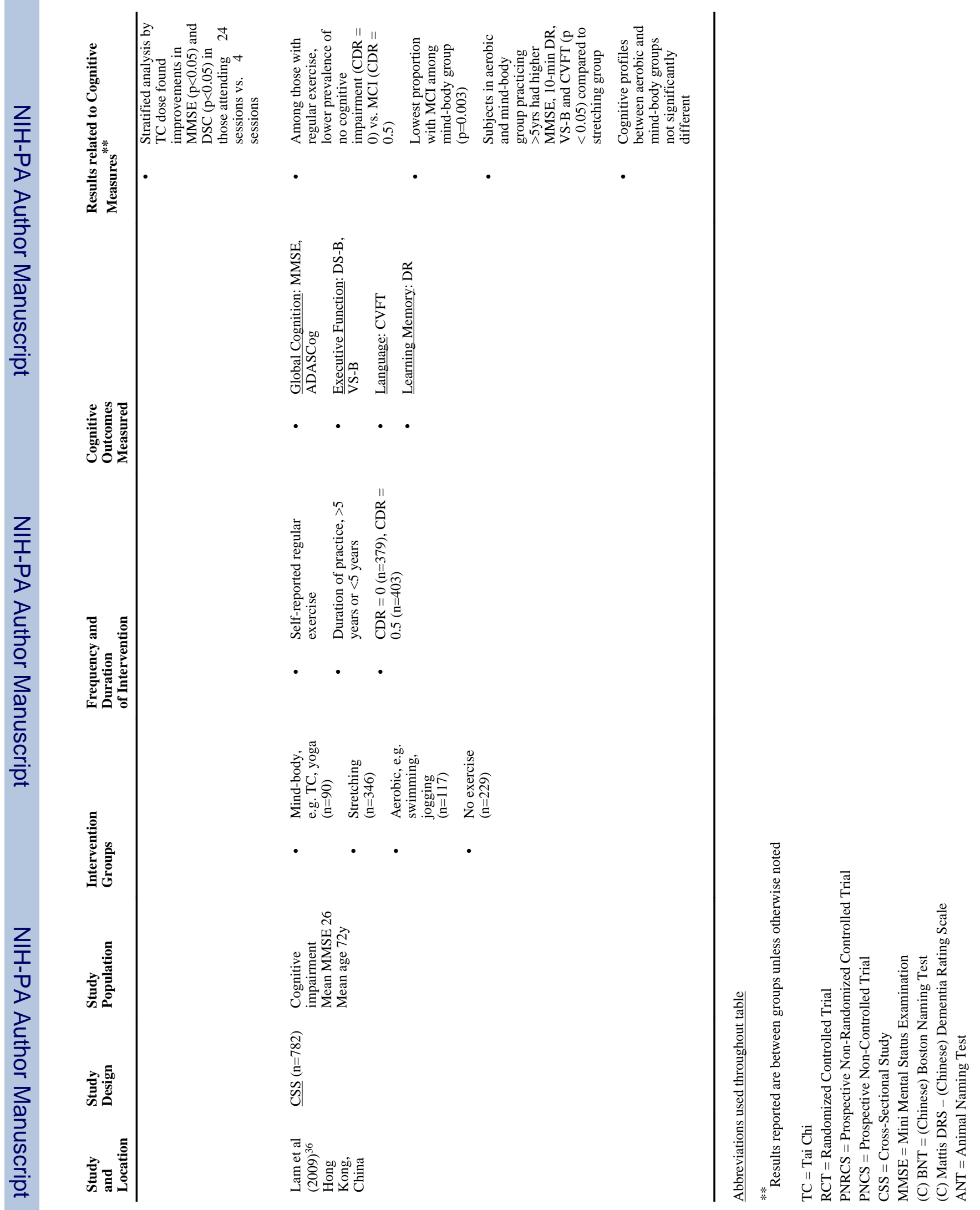




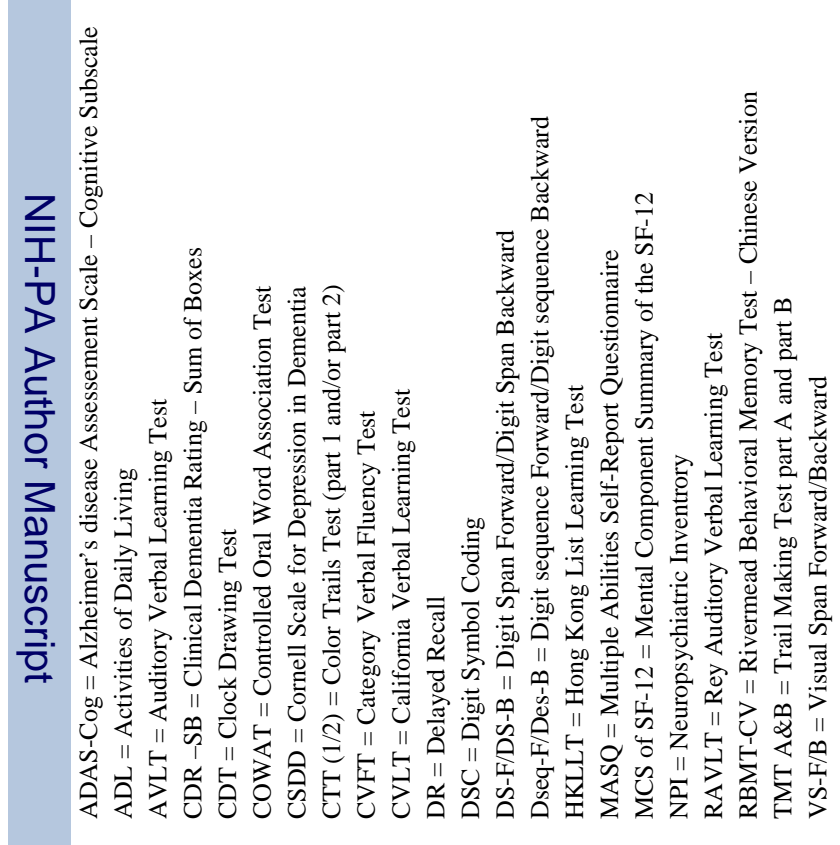




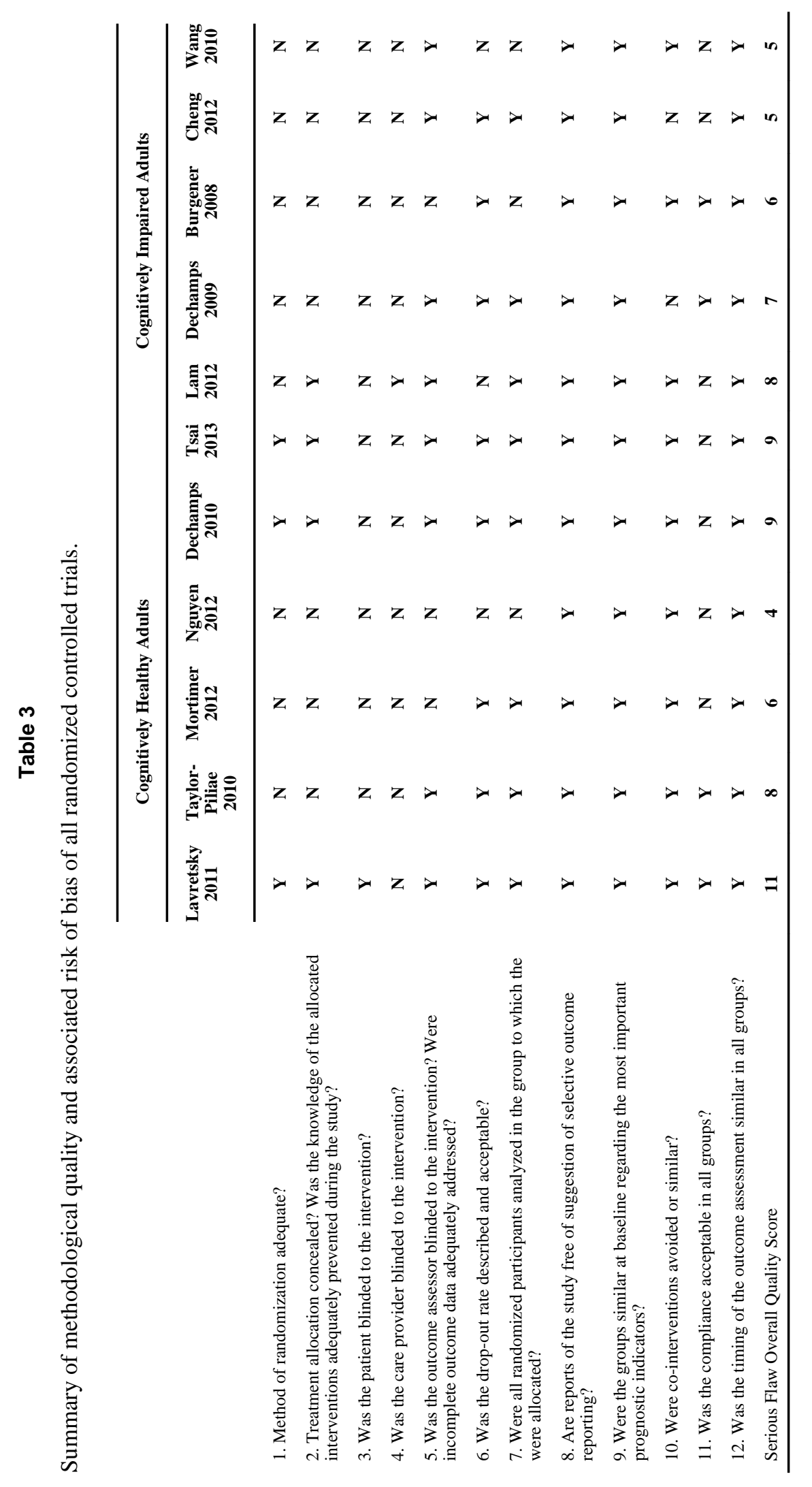

$J$ Am Geriatr Soc. Author manuscript; available in PMC 2015 January 02. 